\title{
Contents Vol. 153, 1967
}

\section{Psychiatric et Neurologia}

Internationale Monatsschrift für Psychiatrie und Neurologie Revue Internationale Mensuelle de Psychiatrie et de Neurologie International Monthly Review of Psychiatry and Neurology

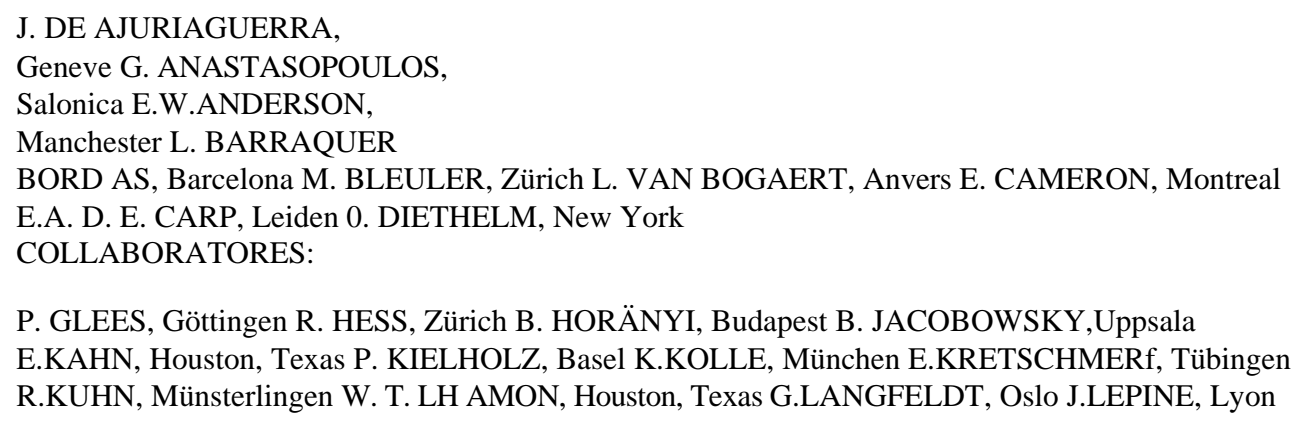

A. LEWIS, London

CHR. MÜLLER, Lausanne

M. MÜLLER, Bern

KURT SCHNEIDER, Heidelberg

P. B. SCHNEIDER, Lausanne

E. SLATER, London

J. STAEHELIN, Basel

H. STECK, Lausanne

G.E. STÖRRING, Kiel

E. STRÖMGREN, Risskov

A. WEBER, Bern

J. WYRSCH, Stans und Bern

EDITOR: J. KLAESI, Schloß Knonau

REDACTOR: E. GRÜNTHAL, Bern

REDACTORES-SECRETARII: N. PETRILOWITSCH, Mainz

TH. SPOERRI, Bern 


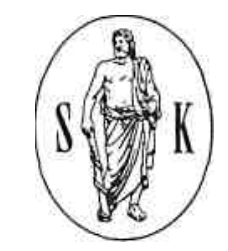

Vol. 153

1967

BASEL (Schweiz)

Alle Rechte» insbesondere das der Übersetzung in fremde Sprachen, vorbehalten. Ohne ausdrückliche Genehmigung des Verlages ist es auch nicht gestattet, diesen Band oder Teile daraus auf photomechanischem Wege (Photokopie, Mikrokopie) zu vervielfältigen.

(C)

Copyright 1967 by S. Karger AG., Basel Printed in Switzerland,

Clichls: Steiner \& Cie., AG., Basel

Druck: Basler Druck- und Verlagsanstalt, Basel

INDEX

BARES, L.:

vide POLAK, 0.

BARTKO, D.; "WAGNEROVÄ, M. and MARSCHALKOVI, L.:

Cerebral Insult and Protein Fractions of the Blood Serum $\quad \ldots . . \quad 128$

BOLZANI, L. et SLTVAR, G.:

Etüde clinique d'un medicament anxiolytique-antidepressif ....

301

Borenstein, P.; GeKiere, F.; Cujo, Ph. et Thouvenot, M.:

Evaluation des effets therapeutiques d'un neuroleptique par la con-frontation des donnees cliniques et paracliniques. A propos d'uneexperience de deux ans de la Tbioridazine.

BreIL, M.A.: $\quad$ Graphologische Möglichkeiten und Grenzen bei der Beurteilung von

Handschriften von Psychopathen

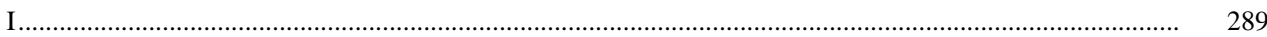

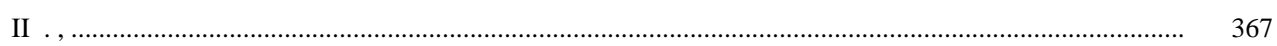

CUJO, PH. : $\quad$ vide BORENSTEIN, P.

FLÜGEL, K. A.: $\quad$ Rezidivierende hysteriforme Psychose (Ganser-Syndrom) bei Syringo-

myelie mit Hydrocephalus .....

GEKIERE, F.: $\quad$ vide BORENSTEIN, P.

GÖMEZ-MARTINEZ, I.:

GROF, P.:

vide VOLAVKA, J.

GRÜNTHAL, E.: . In Memoriam MARTIN REICHARDT

Herbst, A.: $\quad$ vide HitzscheJe, B.

HitzsCHKE, B. und HERBST, A.: 
LAITINEN, L.V.: $\quad$ vide RINNE, U. K.

MALler, O.: $\quad$ Fundamentals for Treatment of Non-organic Chronic Mental Patients 353

MARSCHALKOVÄ, L.: vide BARTKO, D.

MorsiER, G. DE: $\quad$ Les discopathies intervertebrales. Histoire, semiologie, pathogenie, medecine sociale

II .

MRKXAS, L.: $\quad$ vide VOLAVKA, J.

MuCHA, H.: vide MÜNCHOW, $\mathrm{H}$.

MüNCHOW, H. und MuCHA, H.:

Kephalisation des Atlas als Ursache bewegungsabhängiger Störungen

im Arteria-vertebralis-Bereich. $\$ \%$

NYIRÖ, G. f: $\quad$ vide VABGA, E.

OlÄH, I.: $\quad$ vide SZABADI, E.

OOSTERBAAN, W.M.:

Study of a New Drag (Oxazepam) in a Large Psychiatric Institution

PlLIiEM, G.: $\quad$ Bilateraler Ausfall des Gyrus hippocampi und des temporalen Neocortex ohne Klüver-Bucy-Syndrom.

POLÄK, 0. und BARES, L.:

EEG und neurologische Syndrome bei der basilaren Impression . .

Rinne, U.K.: $\quad$ Studies on Myopathy During Corticotrophin Treatment in Multiple

Sclerosis Patients. Multiple

Rinne, U.K.; LAITINEN, L.V. and SONNINEN, V:

Excretion of Homovanillic and Vanilmandelic Acids Before and AfterThalamotomy for

Extrapyramidal Disorders.

SCHERZER, E.: $\quad$ EEG-Veränderungen bei zerebraler Fettembolie

SutVAR, G.: $\quad$ vide BOLZANI, L.

SONNINEN, V.: $\quad$ vide RinNE, U. K.

SPOERRI, TH. : $\quad$ Motorische Schablonen und Stereotypien bei schizophrenen Endzuständen 81

SZABADI, E. and OLAH, I.:

Alterations of the Eyeground Observed in Cases of Panencephalitisnodosa.....

SzOBOR, A.: $\quad$ Die bei Myasthenia gravis vorkommenden psychischen Zustände und psychopathologischen Phänomene

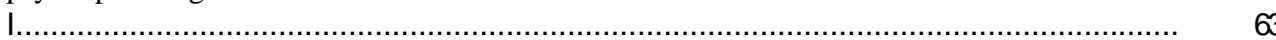

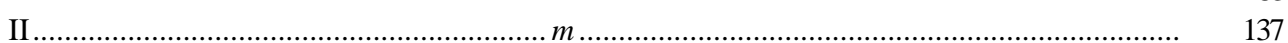

III

Thouvenot, M.: vide Borenstein, P.

VARGA, E. and NYIRÖ, G. f:

A Study of Neurosis in Budapest. A Social-psychiatric Analysis ofClinical Cases Occurring in a Period of 30 Years

VoLAVKA, J.; Grof, P. und MrKLAS, L.:

EEG Frequency Analysis in Periodic Endogenous Depressions . . 384

WAGNEROVÄ, M.: vide BARTKO, D.

WYRSCH, J.: $\quad$ EUGEN KAHN zum 80. Geburtstag

LIBRI .....

\section{E. English Summaries 35}


52

62,125,136,163,176

206

224

230

242

272

306 ,

316

327

335

343

350

365

382

389

409 